$\xi=1$ 圆

\title{
Usability testing on game interface design using video-based behavior analysis
}

\author{
Ibrahim Ahmad ${ }^{1 *}$, Nazreen Abdullasim¹, Norhaida Mohd Suaib² \\ ${ }^{1}$ Universiti Teknikal Malaysia Melaka, Durian Tunggal, 76100 Melaka, Malaysia \\ ${ }^{2}$ Universiti Teknologi Malaysia, 81310 Skudai, Johor, Malaysia \\ *Corresponding author E-mail: ibrahim@utem.edu.my
}

\begin{abstract}
The objective of this study is to quantitatively incorporate user observation into usability evaluation of game interface design. In this study, an experiment was conducted to monitor and record users' behavior using built in video-cam. The experiment was done after the user play "A Garuda" game. All the character movement controlled by user were captured and recorded for comparative analysis. There were about 20 people involved as a subject for this experiment. The data from video recordings were coded with Noldus Observer XT in order to find usage patterns and thus to gather quantitative data for analyzing the GUI effectiveness, efficiency and satisfaction. The result of user's interaction towards the design of game's GUI able to give additional information for the game designer to develop a better responsive game toward its usability. The effect of user's control and emotion that can be seen from user's face will give the information needed to be considered in game development. Previous studies mostly focusing on evaluating the usability with performance measures by only looking at task results. Thus, at the end of this study, a method is proposed by incorporating user observation into usability evaluation of game design interfaces.
\end{abstract}

Keywords: Game design; Usability; User-observation.

\section{Introduction}

Usability is usually understood as a tool for examining User Interface from various perspectives. The usability has been widely used in various level of understanding. One of it is to associate the usability with it efficiency and its effectiveness to the interface. This view was accepted as a theoretical basis for the field of humancomputer interaction.

Many studies on the usability focusing on interface design [1]. The study by [2] for example states that "the difference between the effectiveness of interface and usability is not clear."

Usability has some attributes of its own. The ISO [3] defines usability as "the extent to which a product can be used by specified users to achieve specified goals with effectiveness, efficiency and satisfaction in a specified context of use." While in [4] shows that usability has five attributes: learnability, efficiency, memorability, error rates are low or easy error recovery and satisfaction. In [5] share the same perspective where usability is correct in terms of functionality, efficient to use, easy to learn and remember, error tolerance and subjective matter is made. In addition, in [6] underlines that usability has four factors: usefulness, effectiveness (ease of use), learnability and likeability attitude. This contrasts with [7] which classifies usability into early performance, long-term performance, learnability, retainability, the use of advanced features, the first impression and long-term user satisfaction. Moreover, researchers referred to as a guide in this study is [8]. He underline three criteria; effectiveness, efficiency and satisfaction with the interface application in research.

However, in [9] said that usability is the "extent to which users can successfully learn and use the product to achieve 'a reference to researchers in their research focus in this area".
Usability study conducted may have an impact on research. This is supported by Jeng1who has studied the relationship of interface design with its product acceptance. He himself had stated that the impact of the interface usability study toward the related industry is very large. This is due to the fact that consumer acceptance of a product depends on the usability of the product itself. Based on these facts, this study is based on three construct of the usability aspects. The constructs are based on effectiveness, efficiency and user satisfaction towards the interface design of the computer game produced.

Similar approach also been taken by some of these researcher which are [10-11]. Thus, the basis of this study has taken into account usability aspects as part of the method of determining the suitability of the design characteristics of the game's interface has been selected.

\section{User observation tools}

There are a variety of techniques for usability evaluation including in-depth interviews, think-aloud protocols, scenario building, cognitive walkthrough, heuristic evaluation and surveys [11-12]. However, in conventional methods such as usability testing and surveys, users often do not speak well or express fully. Thus, user observation is necessary for eliciting the detailed information needed.

Referring to the experience of the players during interacting with the game, in [13] uses streaming video to get data for the effectiveness and application efficiency domain tested.

In [14] categorized user observation techniques into 2 types: firstperson and third-person observations. Behavior is self-recorded in first-person observation, while it is recorded from an outside posi- 
tion in third-person observation. Since first-person observation gives understanding in a user's point of view while third-person observation shows related environmental factors, both views need to be considered.

In our experiments, Noldus Observer XT, a video coding software was used to process the video recordings and to gather quantitative data.

\section{Development metrics}

Attributes and factors of usability have been defined in various ways by existing standards and models. Most of them include common attributes for defining usability: effectiveness, efficiency and satisfaction. Definitions for the three factors are described in Table 1 .

Studies have focused on developing methodologies and measurements for evaluating usability in terms of effectiveness, efficiency and satisfaction. Quantitative metrics are necessary in order to analyse data more objectively. This study developed metrics for each factor, while also adopting relevant measures. The metrics for effectiveness and efficiency as shown in Table 2 and 3 respectively were developed to produce results based on data from monitoring finger and eye movements. For satisfaction, relevant subjective criteria were organized for questionnaire as shown in Table 4.

Table 1: Factors of usability (ISO 9241-11, 1998)

\begin{tabular}{c|c}
\hline Factor & \multicolumn{1}{c}{ Definition } \\
\hline Effectiveness & $\begin{array}{l}\text { The accuracy and completeness with which users achieve } \\
\text { goals } \\
\text { Efficiency }\end{array}$ \\
The resources expended in relation to the accuracy and \\
completeness with which users achieve goals \\
The freedom from discomfort and positive attitudes to- \\
wards the user of the system
\end{tabular}

Table 3: Metrics for evaluation of efficiency

\begin{tabular}{cc}
\hline Metric & Description \\
\hline Task completion time & Total time to task completion \\
Deviation from optimal & Number of extra actions taken \\
Number of fixations & Total number of fixations \\
Scanpath length & Total length of scanpath \\
\hline
\end{tabular}

Table 4: Metrics for evaluation of satisfaction

\begin{tabular}{cc}
\hline Metric & Description \\
\hline Task completion time & Total time to task completion \\
Deviation from optimal & Number of extra actions taken \\
Number of fixations & Total number of fixations \\
Scanpath length & Total length of scanpath \\
\hline
\end{tabular}

\section{Experimental design}

An experiment was conducted for usability evaluation of game design based on user observation. Twenty people ( 8 males and 12 females) participated by playing the given game. While the subjects playing the games, the video recorder will record their faces along with the game interface they are playing without them being aware. Whether they lose or win in the game, the recording will be taken into account from the game until the game ends.
According to [15], the use of video recording automatically by the computer has indirectly been able to record every game interface design space played by the user in real time without disturbing their focus while playing the game.

This video recording technique was used by some researchers such as [16-18], while the use of screen footage is intended to see the exploration or exploration activities of users used by researchers such as [19-20].

\subsection{Device and tools}

There are three basic things were used in this study; 1) laptop / PC equipped with camera, 2) "A Garuda" game and also 3) screen recorder software. The camera used will be connected directly to the screen recorder software to record the player's face and also record the game interface screen. Screen recorder software used in this study is Lotus Screen Cam 8.

The features available in Lotus Screen Cam 8 software enable computer screen recording and video recording simultaneously. This recording will be stored in a video file containing both the recording. While using this computer game interface design, the video recording display will be minimized so that the recording of the video will not be seen on the screen.

Video recording and game interface screenshots are shown in Figure 1. The game's interlayer screen recording will see the movement of game characters controlled by the subject. Each movement and character activity will be reflected on the video recording.

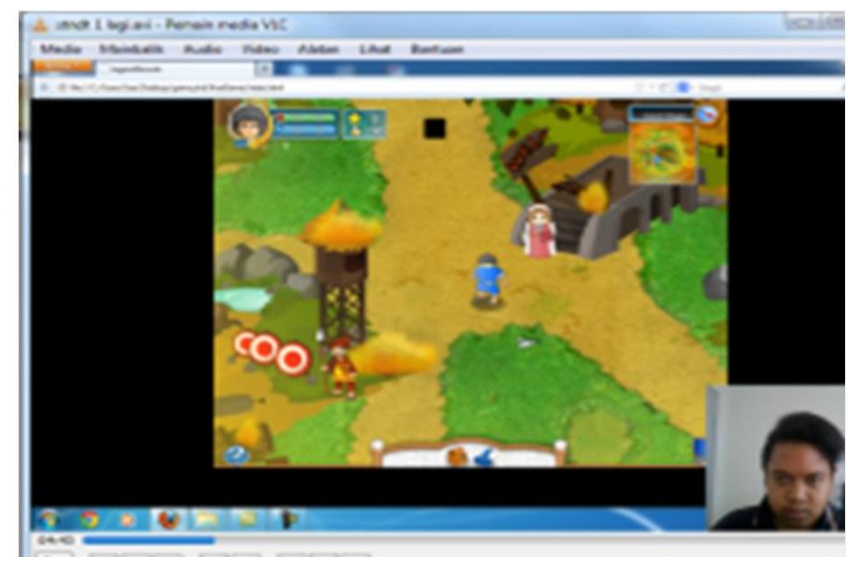

Fig. 1: Example of an image with acceptable resolution

In this study, all the subjects will be given a prototype of the game that has been developed specifically for this purpose. As users interact with the games, video recording and screen recording software are pre-installed before the user starts the game. This is due to the overall recording required to facilitate the process of analysing user activity. The use of Lotus Screen Cam 8 software that can record both types of recording makes it easy to make the recording.

After the recording process is done, the process of analyzing the data will be executed. The process of analyzing this is done using The Observer XT version 9.0 software where the recording will be played back from the beginning of the recording until the user finishes their game. From this moment, face-to-face user interface will be researched and matched with facial express database introduced by [21]. Every emotional impression that can be detected from facial expressions will be highlighted in the software according to the category of emotional effects. The same process will be performed on every video recording that has been recorded on another user.

After all the video recordings were analysed, the determination of the mean value and standard deviation is used to determine the emotional impact with the results of the user interaction interface design used in the game. 


\subsection{Gathering data}

Video recordings were coded into quantitative data using Noldus Observer XT, a software for accurate analysis of observational data. Each subject-controlled character controlled activity will be marked in the Observer software through the recorded video, which will then be analyzed. The recording is a manifestation recorded along with a corresponding time dimension and description on behavioral characteristics.

\section{Results and discussion}

\subsection{Description of game character control and user fa- cial expression}

Based on Figure 1 shown, shows how both footage is screen recording and user face recording is obtained in one video view for analysis purposes using The Observer. Each character movement will be marked to see how often users control game activity based on the checklist that has been set up

\subsection{Result}

Data regarding the usability evaluation of the game interface design is obtained through a video file containing screen recording and recording of the user's face expression during play. In addition, the data log file of every user recorded by the computer using The Observer XT software is also used to support the findings obtained through screen recording and video recording of user faceto-face analysis. To get relevant usability assessments, all users involved in this study analyzed the data. Based on that data source, the usability rating used by the user when using the game interface design is based on the following three factors:
i. Effectiveness facto
ii. Efficiency factor
iii. Satisfaction factor

Table 5: The frequency distribution of activity for the evaluation of effectiveness

\begin{tabular}{c|c|c}
\hline \multirow{2}{*}{ Metrics } & \multicolumn{2}{|c}{$\%$} \\
\cline { 2 - 3 } & Male & Female \\
\hline Task completion rates & 65.39 & 67.56 \\
Provision area & 71.06 & 79.89 \\
\hline
\end{tabular}

Table 6: The frequency distribution of activity for the evaluation of effectiveness

\begin{tabular}{|c|c|c|}
\hline \multirow[b]{2}{*}{ Metrics } & \multicolumn{2}{|c|}{$\%$} \\
\hline & Male & Female \\
\hline $\begin{array}{c}\text { Task completion (number of successful) } \\
\text { Deviation from optimal }\end{array}$ & 4 & 3 \\
\hline a. Press button"RUN" & 4 & 30 \\
\hline b. Meet a enemies & 86 & 60 \\
\hline Scanpath length (average) & $15: 32: 40$ & $18: 02: 34$ \\
\hline
\end{tabular}

Table 7: The frequency distribution of activity for the evaluation of efficiency

\begin{tabular}{c|c|c}
\hline \multirow{2}{*}{ Metrics } & \multicolumn{2}{|c}{$\%$} \\
\cline { 2 - 3 } & Male & Female \\
\hline Error frequency & & \\
a. Press a choice button & 28.94 & 20.11 \\
b. Follow a wrong path & 34.61 & 32.44 \\
\hline
\end{tabular}

Table 8: The frequency distribution of activity for the evaluation of satisfaction

\begin{tabular}{c|c|c}
\hline \multicolumn{2}{|c}{ faction } \\
\cline { 2 - 3 } Metrics & Male & Female \\
\hline Sense of control & 102 & 54 \\
Perception of interaction & 154 & 83 \\
Physical Discomfort & 182 & 73 \\
Enjoyableness & 156 & 69 \\
\hline
\end{tabular}

Table 9: The frequency distribution of activity for the evaluation of satisfaction

\begin{tabular}{c|c|c}
\hline \multirow{2}{*}{ Metrics } & \multicolumn{2}{|c}{$\%$} \\
\cline { 2 - 3 } & Male & Female \\
\hline Total & 88 & 93 \\
\hline
\end{tabular}

Based on Table 5, it is found that the rate of job settlement rate for female users is slightly higher than that of male users. In terms of space accuracy metrics - female users also show a slightly higher percentage of frequency than male users. Whereas in terms of metrics of frequent mistakes made by users, male users often do two things that often include pressing the selected option while fighting and following the wrong path during exploration.

From the analysis of data made in Table 6 and 7, it can be concluded that most of users, especially female consumers, prefer to press the "RUN" button which means to avoid risking losing the game while fighting against the enemy. The frequency of pushing this button is done thus forming a pattern where female users will try to play in a safe state without risking it. This is in contrast to male players, though on average they can spend the game successfully within a short period of time, but the frequency of escaping from taking the risk of losing is very little. In terms of task completion metrics that have successfully completed the game by achieving the goal, the number of successful male users is more than female users.

The analysis results from Table 8 and 9, can be summarized that more male users show satisfaction elements in terms of usability, interaction perceptions and excitement. Even from the physical discomfort criteria, male users also show the frequency of play compared to female users.

\section{Conclusion}

In the experiment, user behavior was closely monitored by recording user interaction with the generated game interface. By coding the video data, quantitative analysis was conducted to evaluate usability with metrics developed.

A comparative evaluation showed that the resulting game interface design can be seen from the effective, efficient and satisfactory angle of user interacting with it. With that a game designer can take this initiative to understand the needs of target users in designing their game interface later.

This study proposed a method for incorporating user observation into usability evaluation of game design interfaces. It can be expected that the method of quantitative analysis based on user observation can contribute by suggesting a way for comprehensive evaluation of usability.

\section{References}

[1] Jeng J (2005), Usability assessment of academic digital libraries: Effectiveness, efficiency, satisfaction, and learnability. Libri 55 , 96-121.

[2] Kim K (2002), A model of digital library information seeking process (DLISP model) as a frame for classifying usability problems. $\mathrm{PhD}$ thesis, Rutgers University.

[3] International Organization for Standardization (2000), ISO I. 9241 9 Ergonomic requirements for office work with visual display terminals (VDTs)-Part 9: Requirements for non-keyboard input devices (FDIS-Final Draft International Standard), ISO.

[4] Nielsen J (1994), Usability engineering, Elsevier.

[5] Kitalong KS (2003), Designing Web sites that work: Usability for the web (book reviews). Technical Communication 50, 98-100.

[6] Booth P (2014), An introduction to human-computer interaction (psychology revivals), Psychology Press.

[7] Hix D \& Hartson HR (1993), Developing user interfaces: Ensuring usability through product and process, John Wiley and Sons.

[8] Frøkjær E, Hertzum M \& Hornbæk K (2000), Measuring usability: are effectiveness, efficiency, and satisfaction really correlated? Proceedings of the SIGCHI Conference on Human Factors in Computing Systems, pp. 345-352. 
[9] Clairmont M, Dickstein R \& Mills V (1998), Testing for usability in the design of a new information gateway. https://arizona.openrepository.com/bitstream/handle/10150/615191/ Testing+for+Usability+in+the+Design+of+a+New+Information+Gatew ay+(web+page).pdf;jsessionid=389385CF96D1DFF116617BD92C 75D621? sequence $=1$.

[10] Dyck J, Pinelle D, Brown BA \& Gutwin C (2003), Learning from games: HCI design innovations in entertainment software. Graphics Interface 2003, 237-246.

[11] Gowases T, Bednarik R \& Tukiainen M (2008), Gaze vs. mouse in games: The effects on user experience. http://citeseerx.ist.psu.edu/viewdoc/download;jsessionid=D10E158 EF73027FF4496C4CBA07EA185?doi=10.1.1.144.1639\&rep=rep1 \&type $=$ pdf.

[12] González MP, Lorés J \& Granollers A (2008), Enhancing usability testing through datamining techniques: A novel approach to detecting usability problem patterns for a context of use. Information and Software Technology 50, 547-568.

[13] Paymans TF, Lindenberg J \& Neerincx M (2004), Usability tradeoffs for adaptive user interfaces: Ease of use and learnability. Proceedings of the 9th International Conference on Intelligent User Interfaces, pp. 301-303.

[14] Lee YS, Hong SW, Smith-Jackson TL, Nussbaum MA \& Tomioka K (2005), Systematic evaluation methodology for cell phone user interfaces. Interacting with Computers 18, 304-325.

[15] Alessi SM \& Trollip SR (2000), Multimedia for learning: Methods and development, Allyn and Bacon Inc.

[16] Chanel G, Ansari-Asl K \& Pun T (2007), Valence-arousal evaluation using physiological signals in an emotion recall paradigm. Proceedings of the IEEE International Conference on Systems, Man and Cybernetics, pp. 2662-2667.

[17] Al Mahmud A, Mubin O, Shahid S \& Martens JB (2008), Designing and evaluating the tabletop game experience for senior citizens. Proceedings of the 5th Nordic Conference on Human-Computer Interaction: Building Bridges, pp. 403-406.

[18] Nacke L (2009), Affective ludology: Scientific measurement of user experience in interactive entertainment. $\mathrm{PhD}$ thesis, Blekinge Institute of Technology.

[19] Sykes J \& Brown S (2003), Affective gaming: Measuring emotion through the gamepad. Proceedings of the ACM CHI'03 Extended Abstracts on Human Factors in Computing Systems, pp. 732-733.

[20] Blewitt W \& Ayesh A (2009), Implementation of Millenson's model of emotions in a game environment. Proceedings of the Artificial Intelligence and Simulation of Behaviour Symposium: AI and Games, pp. 75-80. 